\title{
Keep A Hot Bath
}

\section{Wei Li}

Department of Electronic and Communication Engineering, North China Electric Power University, Baoding 071003, China;

liwei5240@qq.com

Keywords: heat-balance model; dynamic heat-balance model .

\begin{abstract}
There are two basic models built in this paper to keep a hot bath when having a shower in the bathtub: fundamental heat-balance model and dynamic heat-balance model. A reasonable strategy to keep water temperature approximately constant and well-distributed is found in the former. The influence on water temperature due to the existence of human is discussed in the latter. Finally, the feasibility of our models is validated and non-technical explanation of strategy is given.
\end{abstract}

\section{Introduction}

Bathtub is a water pipe device which is use to bath. It is usually in the bathroom. All the time, most of bathtubs are rectangular shape. In recent years, with Acrylic heating bathtub gradually popularized, there are beginning to appear different shapes of the bathtub in people's home. A majority of bathtubs have overflow gate at the bottom of the bathtub. There is also an overflow gate in the top of bathtub.

General specification for bathtub:1700mm in length,800mm in width,700mm in height. General capacity is 230L to 320L.When people is in the bathtub, the water should drown the people's shoulder. If the bathtub is too small, in which people would be not comfortable. If the bathtub is too big, there is a sense of floating instability. The height of the overflow gate determines the height of the water capacity.

Bath without heater heating function can only take heating water to keep the temperature. The lower water temperature is caused by many aspects. There are three main aspects: the heat of water flow changes $\left(Q_{1}\right)$, heat of evaporation of water $\left(Q_{2}\right)$ and Heat conduction $\operatorname{loss}\left(Q_{3}\right)$. The shape and volume of the bathtub and the insulation performance of the bathtub's wall can also affect the heat preservation effect. In the case of adding hot water constantly, water temperature and water flow speed become the key to heat preservation.

\section{Model Establishment}

\subsection{Fundamental heat-balance model}

\section{Step 1. The analysis of its affected factors}

We take the following factors into account, which will affect the water temperature. They are Ambient temperature (A), The heat loss due to heat conduction phenomena (T), Heat transfer between human body and water $(\mathrm{H})$, Water carried by the overflow may take away the heat $(\mathrm{W})$ and Diffusion motion of water molecules (D).

We determine the weights via the Analytical Hierarchy Process (AHP) [Saaty1982]. We build matrix reciprocal matrix by pair comparison:

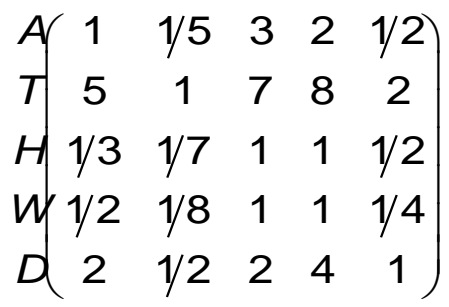

According to the matrix above, after normalization, we get the feature vector 


$$
\omega=[0.135,0.510,0.071,0.062,0.222]^{T}
$$

We can easily get the corresponding weights of five factors. We can safely come to the conclusion that: the proportion of ambient temperature, heat transfer loss and diffusion of water molecules are close to $90 \%$. So we view the above three factors as the control of the water temperature of the judge standard.

\section{Step 2.Take an element}

Based on the above assumptions, we can know that the water temperature in the bath is evenly distributed. We use horizontal one-dimensional model to study. The water in the bathtub is made up of a number of isothermal surfaces.

We remove a micro body from the bathtub along the high surface layer. The bathtub's temperature change is the result of internal and external heat exchange in the water body.

Internal heat exchange in the water body includes: Heat exchange due to the flow of water level direction and Heat exchange due to the flow of water vertical direction(Bath depth is relatively small, so we ignore it).

External heat exchange in the water body includes: Heat absorbed by evaporation of water surface, Heat conduction loss and Water and the tub wall heat transfer.

\section{Step 3. Heat exchange caused by horizontal flow of water $Q_{1}$}

Per unit time, the flow of water is $q_{i}$ from the faucet to the bathtub. And from the water overflow, the flow is $q_{o}$ According to the formula of heat calculation,

The heat brought in per unit time $Q_{i}$ is,

$$
Q=c m \Delta t
$$

$$
Q_{i}=c \rho q_{i} T_{i}
$$

Because we keep the water temperature as close to the initial temperature as possible, we believe that the overflow water temperature approximates to the temperature of the water bath. Per unit time, the heat brought out $Q_{o}$ is,

$$
Q_{o}=c \rho q_{o} T_{o}
$$

Therefore heat exchange caused by horizontal flow of water $Q_{1}$ is,

$$
Q_{1}=c \rho q_{i} T_{i}-c \rho q_{o} T_{o}
$$

\section{Step 4. Heat absorbed by evaporation of water surface $Q_{2}$}

We get the quality that can be expressed as,

$$
m=\rho_{s} E
$$

According to Association for Engineering Construction Standardization standard, indoor open water surface evaporation is expressed as,

$$
E=A(0.00017+0.00013 v)\left(P_{w}-P_{r}\right) \frac{B}{B^{r}} \times 10^{3}
$$

Therefore the heat absorbed by evaporation of water surface $Q_{2}$ is,

$$
Q_{2}=c \rho_{s} A(0.00017+0.00013 v)\left(P_{w}-P_{r}\right) \frac{B}{B^{r}} \times 10^{3}\left(T-T_{s}\right)
$$

\section{Step5.Heat conduction loss $Q_{3}$}

The heat conduction loss of this model includes a heat conduction from water surface and heat conduction from bathtub wall. When there is a temperature difference, heat exchange through conduction is at the interface. According to Fourier Law, the thermal conductivity is in proportion to 
the temperature gradient difference of these substance. Thus, we can get $Q_{3}$, which includes the heat loss of the water heat conduction and the heat loss of heat bathtub wall conduction.

$$
Q_{3}=A_{1} \lambda_{1} \frac{T-T_{s}}{\delta_{1}}+A_{2} \lambda_{2} \frac{T-T_{s}}{\delta_{2}}
$$

\section{Step 6. Heat balance}

We apply the law of conservation of energy, we get the formula as follow,

$$
Q_{1}-Q_{2}-Q_{3}=0
$$

\section{Step7.Conclusion is applied to the model of bathtub}

Under assumptions, we believe that the bathtub shape and capacity primarily affect the amount of water and temperature regulation by affecting the water surface area. According to the formula (10), the unrelated variables in the formula are instead by some constants after calculation. We get,

$$
q_{i} T_{i}=0.0468+0.1577 \mathrm{~A}
$$

\subsection{Dynamic heat balance model}

\section{Step 1. Heat absorbed by evaporation of water surface $Q_{4}$}

We consider how the model depends upon the shape/volume/temperature of the person in the bathtub. We look back how the human affect our model. Because of the existence of man, it may cause changing the area of the evaporation. Because the water temperature is close to the human body temperature. We ignore heat exchange between human body and water(as the above assumption).We set up an unknown quantity a which is express the people occupying the area of the water surface of the bathtub. At this time, the amount of evaporation $E^{\prime}$ is,

$$
E^{\prime}=(A-a)(0.00017+0.00013 v)\left(P_{w}-P_{r}\right) \frac{B}{B^{\prime}} \times 10^{3}
$$

According to formula (12) the evaporation heat which has taken human into account is,

$$
Q_{4}=c \rho_{s} E^{\prime}\left(T-T_{s}\right)
$$

\section{Step 2.Heat conduction loss}

According to the formula (13), we can get heat loss through surface heat transfer and through the wall of the bathtub,

$$
Q_{5}=(A-a) \lambda \frac{\left(T-T_{s}\right)}{\delta}+A_{1} \lambda_{1} \frac{\left(T-T_{s}\right)}{\delta_{1}}
$$

\section{Step3.Body heat loss $Q_{6}$}

We regard the bathtub and the people as a whole. Heat transfer of the human will reduce some of the heat. In the process of human body heat sink, we list the body heat-balance equation. The heat loss caused by the person $Q_{6}$ is:

$$
Q_{6}=Q_{v}+Q_{u}
$$

Lung heat loss can be expressed as:

$$
Q_{v}=0.143-0.00127 T_{a}-0.016 e_{a}
$$

External heat loss of human body can be expressed as:

$$
Q_{u}=\frac{T_{b}-T}{R_{s}+R_{a}}+\frac{e_{b}-e_{a}}{Z_{s}+Z_{a}} \times \frac{R_{a}}{R_{a}+R_{s}}
$$

$T_{b}$ is the human body temperature. $R_{s}$ is skin sensible heat transfer resistance. $R_{a}$ is air sensible heat transfer resistance. $Z_{s}$ is skin latent heat transfer resistance. $Z_{a}$ is air latent heat transfer resistance. $e_{b}$ is the water vapor pressure on body. $e_{a}$ is the water vapor pressure in air.

\section{Step 4.New balance}

The new equilibrium equation can be obtained, 


$$
Q_{1}-Q_{4}-Q_{5}-Q_{6}=0
$$

\section{Step 5.Human movement}

The human movement would change the water discharge. Assuming that the amplitude of the human movement, the frequency is constant, the new overflow traffic is,

$$
Q_{7}=c \rho q_{i} T_{i}-c \rho \varepsilon q_{0} T
$$

According to the formula (14) (15) (19), the new heat balance equation is,

$$
Q_{7}-Q_{5}-Q_{6}=0
$$

\section{Step 6.Bubble bath}

We take into account the impact of adding bubble agent on the model will have two aspects.

1. The bubble's density is smaller than water, so the bathtub will be covered with bubble .We know that all objects that are above absolute zero can generate heat radiation. The higher the temperature, the greater the total radiation.

According to the thermal radiation formula:

$$
Q=\varepsilon \sigma(T+273.13)^{4}
$$

Since the water surface is covered with bubbles, the long wave emission rate will be correspondingly smaller, so the bubble bath will reduce the amount of radiation.

2. Bubble bath has an effect on the evaporation coefficient and heat transfer coefficient of water. Adding a bubble will make the evaporation coefficient and heat transfer coefficient smaller. It can be seen from the formula (8) and (9) when evaporation coefficient $\left(p_{w}-p_{r}\right)$ and heat transfer $(\lambda)$ coefficient change small, the amount of heat loss and heat loss of water surface is decreased. Therefore, the bubble bath can play a certain role in heat preservation.

\section{Numerical Computation}

We determine values for some parameters.

Generally determine the specific heat capacity of water to be $4.2 \cdot 10^{3} \mathrm{~J} /\left(\mathrm{kg} .{ }^{\circ} \mathrm{C}\right)$. Density of water is about $1.0 \times 10^{3} \mathrm{~kg} / \mathrm{m}^{3}$.Moreover, according to the room temperature is generally $21^{\circ} \mathrm{C}$, the density of water vapor should be $0.01833 \mathrm{~kg} / \mathrm{m}^{3}$ According to dimensions of an overflow opening and flow rate of drainpipe generally, we assume pouring flow is $q_{o}=0.0012 \mathrm{~m}^{3} / \mathrm{s}$ As the commonsense, the optimal temperature in a bath is and the normal temperature of the human body is about $37^{\circ} \mathrm{C}$.

According the size of a regular bathtub, we assume cross-sectional area of a tub is $1.36 \mathrm{~m}^{2}$.Moreover, when the room temperature is $21^{\circ} \mathrm{C}$, air flow rates is $5.09 \mathrm{~m} / \mathrm{s}$. We conservatively assume atmospheric indoor is $100 \mathrm{kPa}$ and conductivity of water is $602 \mathrm{mw} /\left(\mathrm{m} \cdot{ }^{\circ} \mathrm{C}\right)$. We define the temperature of water vapor as $30^{\circ} \mathrm{C}$ according assumption before.

On the basis of data above, we get expression of heat exchange resulting from horizontal water flow. We can easily calculate the Water-surface Evaporating Capacity is 5.1719J. Thus, we get absorption of heat due to water surface evaporation is about 398.16J.Moreover, we can calculate conductive heat-loss is $368.424 \mathrm{~J}$ and heat pouring flow take away is about $19650 \mathrm{~J}$.

We analyze and calculate the data above using conservation of energy. Then, we find $q_{i} T_{i}$ is a constant value. Numerical results differed negligibly. As Fig. 1.shows, if cross-sectional area of a tub is given, as long as control $q_{i} T_{i}$ equal to the value, we can keep the water as close as possible to the initial temperature ideally without wasting too much water.

Maintaining other effect factors constant, we can find the dependence between our models and the shape and volume of the tub. As Fig. 2.shows, $q_{i} T_{i}$ increase with cross-sectional area A. That is to say, if the shape and volume increase, A will increase commensurately and heat conservation effects 
will reduce. In such circumstances, we need to increase flow rate or temperature. Therefore, we offer our strategy that a regular not large tub play a role in water conservation.

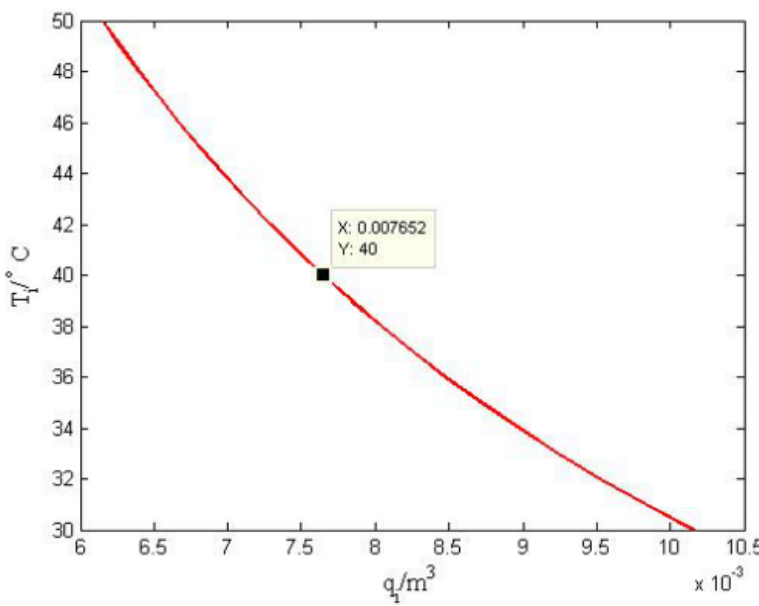

Fig. 1 The relation between $T_{i}$ and $q_{i}$

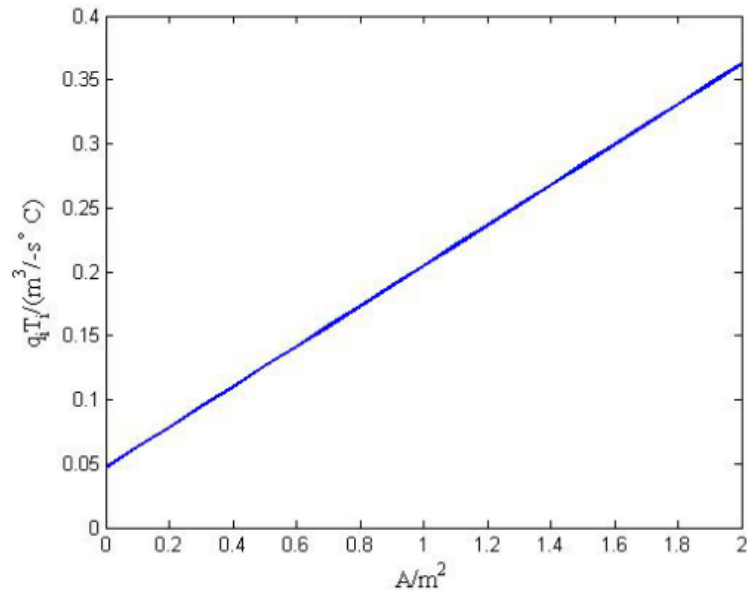

Fig. 2.The relation between $q_{i} T_{i}$ and $\mathrm{A}$

We get a human heat release equation regarding person and tub as a whole. A new heat balance equation can be got according absorption of heat due to water surface evaporation and conductive heat-loss. By calculate, we find the relation between $q_{i} T_{i}$ and person' s occupied area. As Fig. 3 shows, we arrive at the conclusion that the more a person's occupied area, the less according absorption of heat. However, human heat release accelerates the drop of temperature.

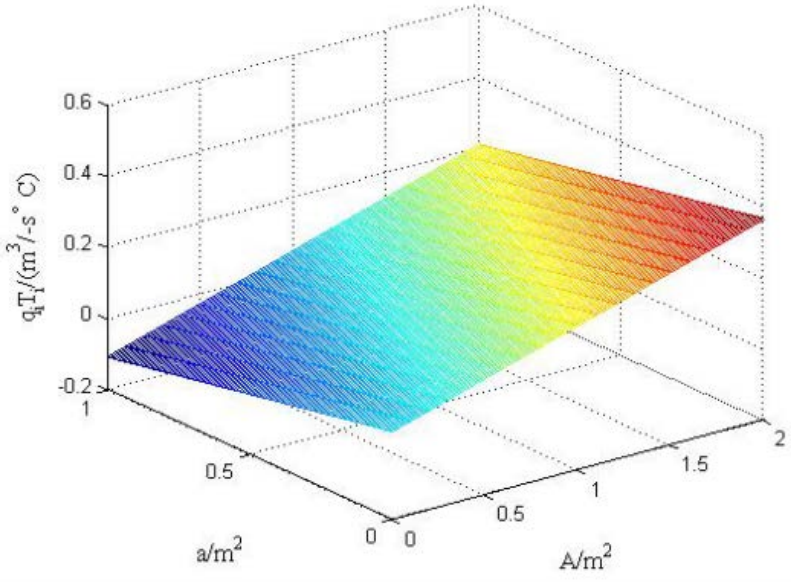

Fig. 3.The relation between $q_{i} T_{i}$, a and A

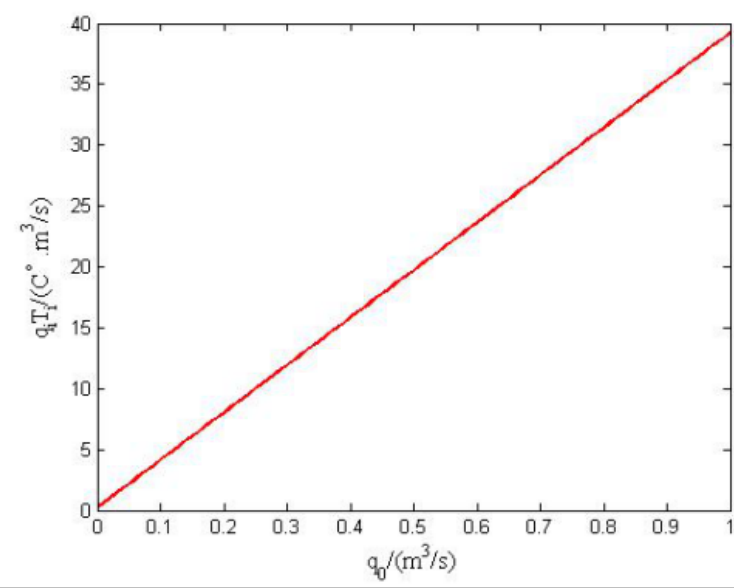

Fig. 4. The relation between $q_{i} T_{i}$, a and $\mathrm{q}$

According to the data above, we find the relation between $q_{i} T_{i}$ and pouring flow under the motions made by the person. As Figure4.shows, if the motions increase in intensity, the person requires higher temperature

or velocity.

\section{Local Optimization}

We re-examine the parameters for formula, then we got water radiant heat equation:

$$
Q_{s}=\varepsilon_{w} \cdot \sigma \cdot(T+273.13)^{4}
$$

Where $\sigma$ is the Stefan-Boltzmann constant,

$$
\sigma=117 \times 10^{-4} \mathrm{kcd} / \mathrm{m}^{2} \cdot \mathrm{d} \cdot \mathrm{k}^{4}
$$

And $\varepsilon_{w}$ is surface long wave emissivity,

$$
\varepsilon_{w}=0.03
$$

Relationship between the density and the temperature $\mathrm{T}$ can be expressed as 


$$
\rho=\rho_{o}+a T^{2}+b T
$$

Meanwhile, $\mathrm{a}=-0.0067, \mathrm{~b}=0.07$. Finally, our new equation is calculated as follow,

$$
C\left(\rho_{o} T_{i}+a T_{i}^{3}+b T_{i}^{2}\right) q-C\left(\rho_{o} T+a T^{3}+b T^{2}\right) q_{o}-a m \Delta T-A \lambda \frac{T-T_{S}}{\delta}-\varepsilon_{w} \sigma(T+273.13)^{4}=0
$$

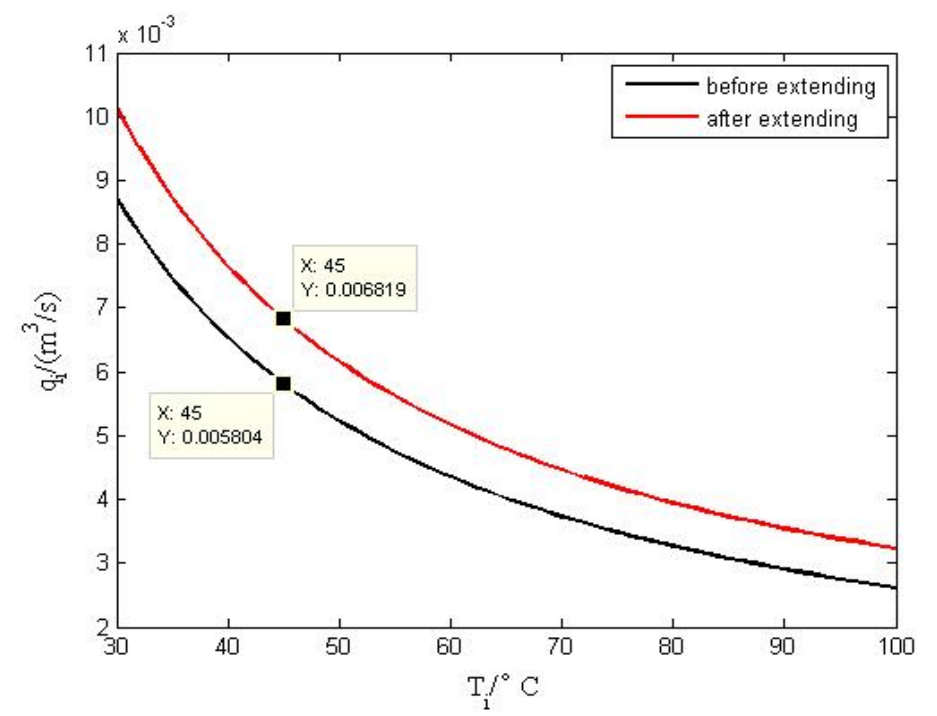

Fig. 5 The compare between the basic model and the extended model

As Fig. 5 shows, the graph is the curve between the influx and the temperature. In the picture, we make a numerical analysis of the curve in the model one and the curve in the optimization model .And take into account the appropriate temperature of the human body and try not to waste too much water. We determine the optimal scheme: water inlet temperature is 45 degrees, the water flow rate is $0.006819 \mathrm{~m}^{3}$.

\section{Summary}

1. The fundamental strengths of our model is that we take various factors into consideration. It enhances the feasibility of model.

2. The numerical computations are precise.

3. Our model has few input parameters, leading to good robustness and sensitivity.

4. The small part of the data is given by the model maker. It has a certain deviation.

\section{References}

[1] Lee S W, Tai Y C. A micro cell lysis device[J]. Sensors and Actuators A: Physical, 1999, 73(1): 74-79.

[2] Gurtin M E, Pipkin A C. A general theory of heat conduction with finite wave speeds[J]. Archive for Rational Mechanics and Analysis, 1968, 31(2): 113-126.

[3] Planck M. The theory of heat radiation[M]. Courier Corporation, 2013.

[4] Bowen I S. The ratio of heat losses by conduction and by evaporation from any water surface[J]. Physical review, 1926, 27(6): 779 